\section{Integrating genetic and epigenetic factors in chronic myeloid leukemia risk assessment: toward gene expression-based biomarkers}

\author{
Vaidehi Krishnan, 1,2 Dennis Dong Hwan Kim,, ${ }^{2,3}$ Timothy P. Hughes ${ }^{2,4,5,6}$ \\ Susan Branford ${ }^{2,4,7,8}$ and S. Tiong Ong On,2,9,10,11 $^{1,2}$
}

${ }^{1}$ Cancer and Stem Cell Biology Signature Research Program, Duke-NUS Medical School, Singapore, Singapore; '2International Chronic Myeloid Leukaemia Foundation, Bexhill on Sea, UK; ${ }^{3}$ Department of Medical Oncology and Hematology, Princess Margaret Cancer Center, University Health Network, University of Toronto, Toronto, Ontario, Canada; ${ }^{4}$ School of Medicine, University of Adelaide, Adelaide, Australia; ${ }^{5}$ South Australian Health \& Medical Research Institute, Adelaide, Australia; ${ }^{6}$ Department of Hematology, Royal Adelaide Hospital, Adelaide, Australia; ${ }^{7}$ Department of Genetics and Molecular Pathology, Center for Cancer Biology, SA Pathology, Adelaide, Australia; ${ }^{8}$ School of Pharmacy and Medical

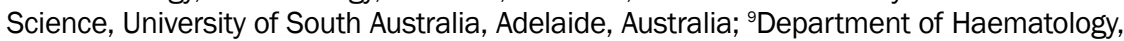
Singapore General Hospital, Singapore; ${ }^{10}$ Department of Medical Oncology, National Cancer Centre Singapore, Singapore and ${ }^{11}$ Department of Medicine, Duke University Medical Center, Durham, NC, USA

\section{ABSTRACT}

$\longrightarrow$ ancer treatment is constantly evolving from a one-size-fits-all towards bespoke approaches for each patient. In certain solid cancers, including breast and lung, tumor genome profiling has been incorporated into therapeutic decision-making. For chronic phase chronic myeloid leukemia (CML), while tyrosine kinase inhibitor therapy is the standard treatment, current clinical scoring systems cannot accurately predict the heterogeneous treatment outcomes observed in patients. Biomarkers capable of segregating patients according to outcome at diagnosis are needed to improve management, and facilitate enrollment in clinical trials seeking to prevent blast crisis transformation and improve the depth of molecular responses. To this end, gene expression (GE) profiling studies have evaluated whether GE signatures at diagnosis are clinically informative. Patient material from a variety of sources has been profiled using microarrays, RNA sequencing and, more recently, single-cell RNA sequencing. However, differences in the cell types profiled, the technologies used, and the inherent complexities associated with the interpretation of genomic data pose challenges in distilling GE datasets into biomarkers with clinical utility. The goal of this paper is to review previous studies evaluating GE profiling in CML, and explore their potential as risk assessment tools for individualized CML treatment. We also review the contribution that acquired mutations, including those seen in clonal hematopoiesis, make to GE profiles, and how a model integrating contributions of genetic and epigenetic factors in resistance to tyrosine kinase inhibitors and blast crisis transformation can define a route to GE-based biomarkers. Finally, we outline a four-stage approach for the development of GE-based biomarkers in CML.

\section{Introduction}

Chronic myeloid leukemia (CML) is a clonal disorder of the hematopoietic stem cell compartment defined and driven by the BCR-ABL1 gene rearrangement and the tyrosine kinase it encodes. ${ }^{1}$ Clinically, it is accompanied by an expansion of mostly myelo-erythroid progenitors that maintain the ability to differentiate terminally into neutrophils. Prior to the introduction of ABL1 tyrosine kinase inhibitors (TKI), most patients would progress to a terminal blast crisis (BC) stage marked by the acquisition of additional genetic abnormalities within an average of 5-7 years. ${ }^{2}$ In this stage, the clinico-pathological features were the inexorable accumulation of either myeloid or lymphoid progenitors that had acquired aber- 
rant self-renewal properties, broad resistance to cytotoxic therapies, and eventual patient demise from bone marrow failure. ${ }^{2}$ The arrival of TKI at the turn of the century resulted in remarkable responses, such that most individuals treated in chronic phase (CP) CML can expect to achieve near-normal life expectancies. ${ }^{3}$ Nevertheless, CML-related deaths are still reported, mainly due to resistance and progression to $\mathrm{BC}$, especially in the first few years of treatment. ${ }^{4}$

\section{Current treatment aims and features of an ideal biomarker}

Current therapeutic aims are directed at achieving sufficiently deep molecular responses that the risks of $\mathrm{BC}$ transformation are effectively negligible and, in the longer-term, increasing the rates of treatment-free remission. ${ }^{5-7}$ Clinical guidelines toward achieving deep molecular responses have been reviewed elsewhere, ${ }^{8}$ and at their core, prescribe the measurement of $B C R-A B L 1$ transcript levels using the International Scale (IS) every 3 months as a readout of the depth of the response to TKI. In turn, the depth of TKI response serves as a critical biomarker guiding patient management and prognostication (Figure 1).

Given current treatment goals, an ideal biomarker would accurately predict patients who will achieve a deep molecular response with first-line TKI, or require a switch to alternative therapy, and, among those who achieve a deep molecular response, those who will be able to stop TKI successfully (Figure 1). The biomarker would be informative from the time of diagnosis and prior to TKI initiation, since this would enable early stratification of patients for therapy with a first-generation versus a second/third-generation TKI, allosteric BCRABL1 inhibitor, a clinical trial, or preparation for allogeneic transplantation. Additionally, among patients who meet the criteria for stopping TKI therapy, the ideal biomarker would identify additional therapies that would enhance treatment-free remissions. Finally, gene expression (GE)-based biomarkers should be clinically robust, and widely available among centers and regions in both low and high Human Development Index countries. ${ }^{9}$

\section{Why gene expression-based biomarkers?}

Contributions from genetic and epigenetic mediators to $\mathrm{TKI}$ resistance and $\mathrm{BC}$ transformation are well documented, ${ }^{10-14}$ and it is axiomatic that genetic or epigenetic factors mediating these outcomes will contribute to a cell's GE signature. Accordingly, GE signatures offer a molecular profile that integrates risk factors encoded by both mutations and epigenetic states. However, faithfully extracting and interpreting GE-based information in clinical settings is challenging. Barriers to adoption include technical limitations, logistical factors, as well as differences in study design and data analysis, and are described below.

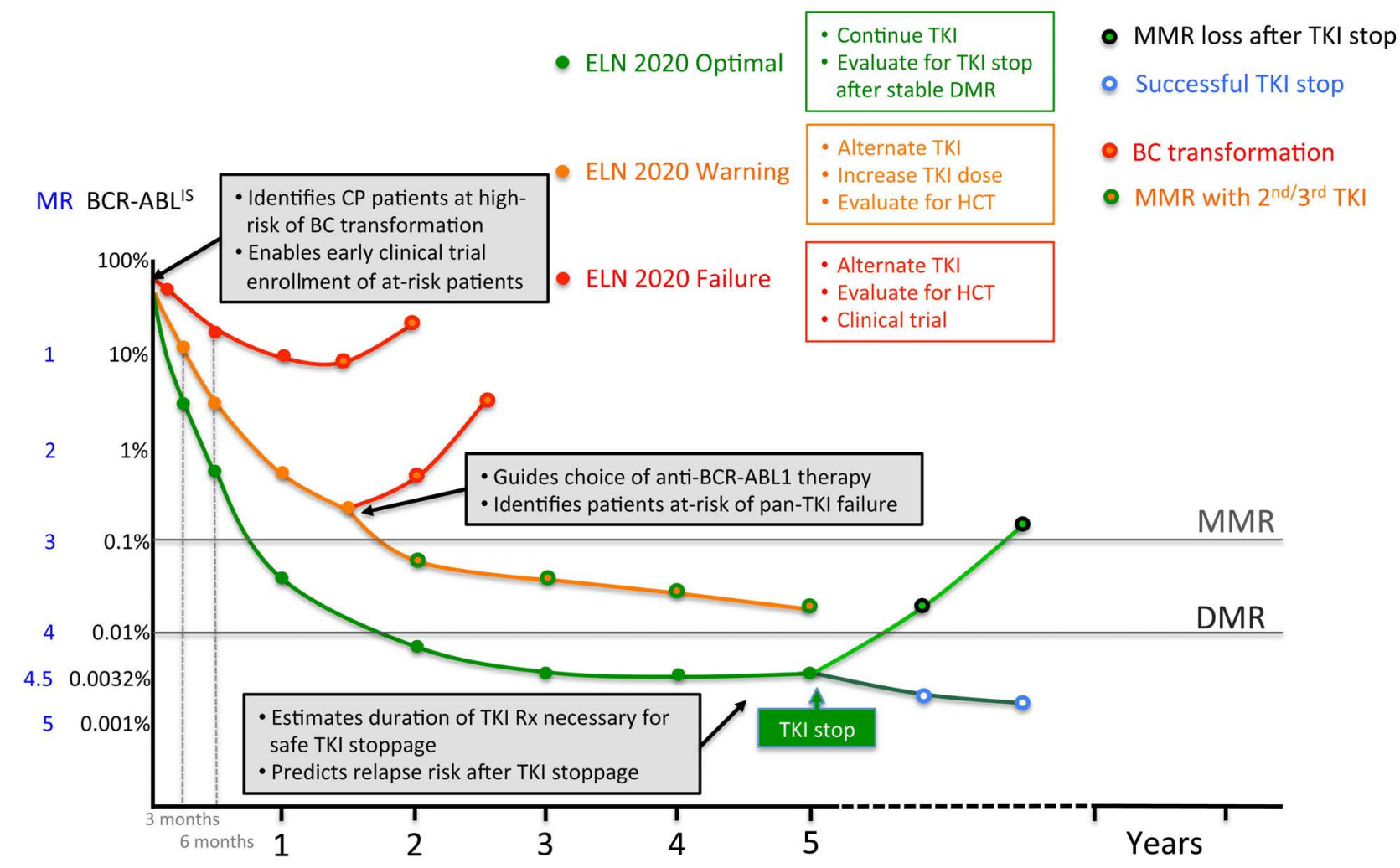

Figure 1. Features of an ideal chronic myeloid leukemia biomarker. Curves indicate changes in $B C R-A B L 1$ transcript levels, meausred using the International Scale (BCR-ABL1 $1^{15}$ ), following initation of tyrosine kinase inhibitor (TKI) therapy in patients with chronic phase (CP) chronic myeloid leukemia (CML). The corresponding molecular response (MR) value is provided next to the BCR-ABL ${ }^{\text {Is }}$ value. Green, orange, and red curves are representative of patients in European LeukemiaNet 2020 'optimal', 'warning', and 'failure' cateogories respectively. Major (MMR) and deep (DMR) molecular remissions are defined as 0.1\% (MR 3) and 0.01\% (MR4) BCR$A B L^{\prime s}$, respectively. Green, orange, and yellow bullet points indicate guidelines for each category of response. Gray boxes describe predictive capabilities of an ideal biomarker. HCT: hematopoietic stem cell transplant. 
Despite the barriers, recent advances in technological and computational platforms are enabling the interrogation of patient samples on an unprecedented scale, and are being translated into robust technical assays on patient material that are reproducible in clinical laboratories. ${ }^{15}$ Such advances may eventually result in the identification of pretreatment biomarkers that not only predict TKI resistance but suggest alternative non-BCR-ABL1-targeting therapies to pre-empt the emergence of clinical resistance. Accordingly, it is timely to review the results of GE studies using primary patient material annotated for clinical outcomes, and assess how genetic and epigenetic factors associated with treatment outcome contribute to GE signatures. In doing so, it is also important to develop models incorporating the interplay between genetic and epigenetic factors, and determine how best to use the resulting GE outputs to understand and predict CML drug resistance and transformation. Finally, it is incumbent on the CML community to outline the practical steps needed for the clinical development of GE-based biomarkers in CML.

\section{Gene expression signatures associated with resistance to tyrosine kinase inhibitors}

Since the beginning of the TKI era, a variety of diagnostic material from CP patients has been used to discover TKI-resistance GE signatures (Table 1). Here, we review the key conclusions from these studies.

\section{Gene expression using peripheral blood}

In the earliest research by Kaneta et al. ${ }^{16}$ and McLean et al. ${ }^{17}$ microarray studies were conducted on blood from imatinib responders and non-responders. Apart from $C B L B$, which was downregulated in responders, there was no overlap between the two datasets. De Lavallade et al. conducted microarray studies on peripheral blood mononuclear cells to identify a 105-gene set that was enriched in imatinib non-responders, comprising mainly genes in cell cycle and DNA repair pathways. ${ }^{18}$ However, the GE signature could be validated only in an imatinibtreated cohort but not in a cohort treated with interferon$\alpha$. As a targeted approach, the expression of 21 genes associated with TKI responses and disease progression was studied by Zhang et al. ${ }^{19}$ Increased PTGS1 expression was the only gene that differentiated primary imatinib-resistant patients from responders, while 15 genes distinguished CP from BC. Twelve genes distinguished imatinib-responsive from secondary imatinib-resistant CML without $B C R-A B L 1$ mutations, of which $L Y N$, JAK2, PTPN22 and CEBPA downregulation was shared with BC samples. The study concluded that at least some features of secondary imatinib resistance overlap with BC transformation.

More recently, Kok et al. conducted microarray-based analysis on diagnostic blood from $96 \mathrm{CP}$ patients from the TIDEL-II trial to predict failure of early molecular response,$^{20}$ which correlates with inferior long-term outcomes. ${ }^{21,22}$ Three hundred sixty-five differentially expressed genes were identified which were enriched for 'cell cycle' and 'stemness' (MYC, HOXA9, $\beta$-catenin) but depleted for 'immune-response' categories in the group with early molecular response failure. A binary classification model was built to predict early molecular response failure based on 17 genes and the signature was validated in an independent cohort. Of these, eight genes IGFBP2, SRSF11, BAX, CDKN1B, BNIP3L, FZD7, PRSS57, and RPS28 intersected with findings of previous CML TKIresistance and progression studies. This study demonstrated that GE information from diagnostic samples could predict events long in the future, including major molecular response (MMR) at 24 months, MR4.5 at 5 years, and $\mathrm{BC}$ transformation.

\section{Gene expression using bone marrow}

Independently, a series of studies used unselected bone marrow for comparisons of GE between groups of patients with different treatment responses. Frank et al. identified a 128 GE signature associated with imatinib resistance, specifically in an interferon- $\alpha$ pre-treated cohort. Differentially expressed genes were involved in apoptosis (CASP9, TRAP1), DNA repair (MSH3, DDB2), oxidative stress protection (GSS, PON2, VNN1) and centrosomes (ID1)..$^{23}$ Villuendas et al. ${ }^{24}$ identified 46 differentially expressed genes of which a six-gene prediction score (BIRC4, FZD7, IKBKB, IL-7R, TNC, VWF) that correlated with imatinib resistance after interferon- $\alpha$ failure developed. Differentially expressed genes were involved in cell adhesion (TNC and SCAM-1), drug metabolism (COX1 or PTGS1), protein tyrosine kinases (MKNK1), and phosphatases (BTK and PTPN22). Notably, the MKNK1/2 kinases have been shown by two independent groups to be involved in $\mathrm{BC}$ transformation. ${ }^{25,26}$ In contrast to the prior studies, Crossman et al. found no differentially expressed genes between the imatinib responder categories. The use of mixed peripheral blood and bone marrow samples, unselected white blood cells and a heterogeneous cohort of patients in late CP and heavily pre-treated, were suggested as potential reasons for the negative results. ${ }^{27}$ The important conclusion was that GE comparisons should be made on purified $\mathrm{CD} 4^{+}$cells. Indeed, in a meta-analysis comparing six published GE studies in CML, DDX11, MSH5, and RAB11FIP3 were the only genes coincident between any two of the studies. ${ }^{28}$ The small differences in differential GE between responder groups, different GE platforms, different statistical methods and different sources of cells profiled were suggested reasons for the poor intersection. The disappointing results from unselected peripheral blood and bone marrow provided the impetus to isolate and study CD34+ fractions.

\section{Gene expression using CD34+ cells}

McWeeney et al. were the first group to use CD34 ${ }^{+}$cells from diagnostic bone marrow. ${ }^{11}$ Cell adhesion genes were upregulated in imatinib-resistant patients suggesting that $\mathrm{CD} 34^{+}$cells may establish more adhesive interactions with the bone marrow milieu. The enrichment for $\beta$ catenin binding targets suggested activated Wnt/ $\beta$-catenin signaling in imatinib-resistant patients, a feature shared with $\mathrm{CD} 34^{+}$progenitors from BC. ${ }^{26,29}$ The authors concluded that primary resistance to imatinib might reflect more advanced disease progression. A 75-probe minimal gene classifier predicted $88 \%$ of responders and $83 \%$ of nonresponders in a validation cohort. Importantly, the authors of this paper compared their GE signatures to those predicting early BC transformation, as discussed below, and provided an important resource for validation and comparison of other CD34+-based GE datasets. 


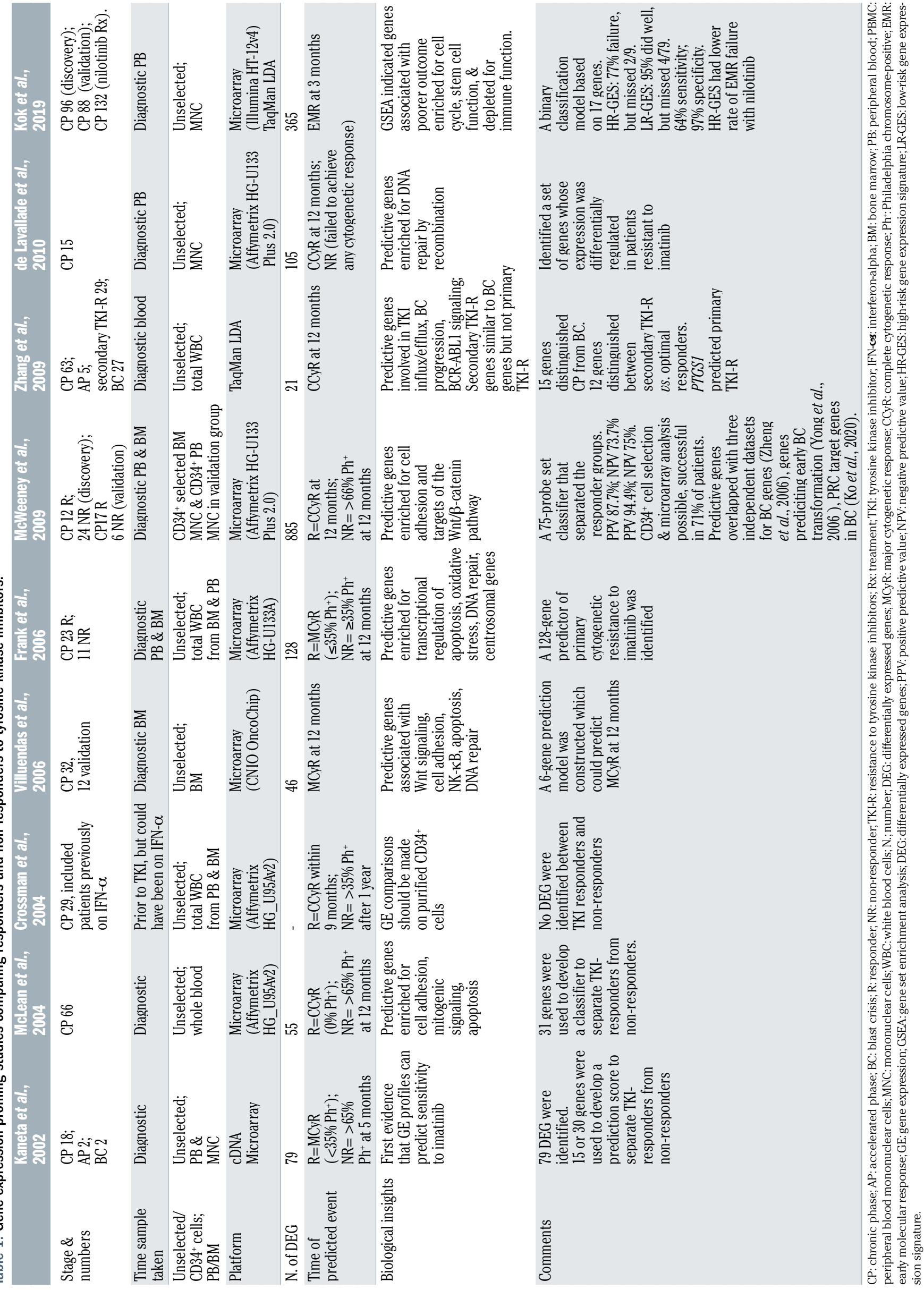




\section{Single-cell-based gene expression analysis}

Recent advances in single-cell analysis have enabled novel GE-based insights on the roles of tumor cell heterogeneity and clonal evolution under the selective pressure of therapeutics, with obvious applications in biomarker development. ${ }^{30}$ Leukemia stem cell (LSC) heterogeneity was characterized by Warfvinge et al. by combining highthroughput immunophenotyping with single-cell GE profiling with a defined panel of genes. ${ }^{31}$ LSC sub-fractions with more primitive and quiescent signatures had a higher persistence after TKI therapy with the most TKI-insensitive population identified as $\operatorname{Lin}^{-} \mathrm{CD} 34^{+} \mathrm{CD} 38^{\text {low }} /-$ CD45RA-cKIT-CD26 stem cells. Giustacchini et al. used the Smart-seq2 platform to combine single-cell RNAsequencing analysis with $B C R-A B L 1$ transcript detection using purified stem cells. A sub-population of BCR-ABL1+ quiescent stem cells enriched for hematopoietic stem cell (HSC) signatures was found to persist during TKI therapy. ${ }^{32}$ Intriguingly, the BCR-ABL1 cells in CML patients were enriched for inflammatory, tumor growth factor- $\beta$ and tumor necrosis factor- $\alpha$ hallmarks and discriminated between the TKI-responder groups.

In addition to LSC-derived signatures, the GE signature of immune cells can be equally instructive. For example, plasmacytoid dendritic cells, the major producers of interferon- $\alpha$ in vivo, promoted resistance to nilotinib in CML patients. ${ }^{33}$ These studies imply that the cytokines released by immune cells in the bone marrow microenvironment, and the transcriptomic changes that they bring about on the LSC, may activate cytokine-dependent TKI resistance programs. ${ }^{34}$ Together, these single-cell studies demonstrate that GE signatures within malignant and nonmalignant compartments in CML are prognostically informative. We anticipate the discovery of additional biomarkers among discrete cell types which have the potential to be assayed by platforms available in standard pathology laboratories, e.g., by flow cytometry or immunohistochemistry.

\section{Gene expression signatures associated with blast crisis progression}

Transcriptomic comparisons between the $\mathrm{CP}$ and $\mathrm{BC}$ stages have uncovered progression-related signatures that can herald BC transformation (Table 2). In the pre-TKI era, the time to $\mathrm{BC}$ transformation from $\mathrm{CP}$ varied between patients, and to understand this difference, Yong et al. compared CD34+ cells from leukapheresis samples provided by patients who progressed to BC within 3 years (aggressive leukemia) or after more than 7 years (indolent leukemia) following diagnosis. ${ }^{13}$ The study identified that lower $C D 7$ with higher $P R 3$ and $E L A 2$ expression at diagnosis was associated with longer survival. Intriguingly, when the GE signatures identified by Yong et al. and McWeeney et al. were compared, a significant overlap was found. ${ }^{11}$ This important study demonstrated that biological processes associated with TKI resistance and early BC transformation overlapped, and that $\mathrm{CD} 34^{+}$cells from different sources (bone marrow vs. peripheral blood) contained this information.

In the post-TKI era, a landmark study by Radich et al. identified distinct transcriptional programs during $\mathrm{BC}$ progression. ${ }^{25}$ About 3,000 genes were associated with the $\mathrm{BC}$ stage with a dysregulated WNT/ $\beta$-catenin pathway, decreased Jun B and FOS, and higher PRAME expression. The Radich dataset was subsequently used to compute a six-gene signature comprising NOB1, DDX47, IGSF2, LTB 4R, SCARB1, and SLC25A3 to predict progression. ${ }^{35}$ Independently, Zheng et al. isolated CD34 $4^{+}$cells and identified 34 differentially expressed genes as cells transited from $\mathrm{CP}$ to $\mathrm{BC}$. Among the misregulated genes, SOCS2 and CD52 were downregulated while HLA-related genes were overexpressed in BC. ${ }^{36}$

To understand the biological mechanisms underlying GE changes in TKI resistance and $\mathrm{BC}$, a recent study tested the hypothesis that prognostically important genes were enriched for targets of the polycomb repressive complex (PRC; see below). ${ }^{10}$ Importantly, target genes of PRC-asso-

Table 2. Gene expression profiling studies comparing chronic phase and acute phase.

\begin{tabular}{|c|c|c|c|c|c|}
\hline & $\begin{array}{l}\text { Yong et all, } \\
2006\end{array}$ & $\begin{array}{l}\text { Radich ot al., } \\
2006\end{array}$ & $\begin{array}{l}\text { Zheng et al., } \\
2006\end{array}$ & $\begin{array}{l}\text { Oehler et al., } \\
2009\end{array}$ & $\begin{array}{l}\text { Ko et all, } \\
2020\end{array}$ \\
\hline Stage \& numbers & СР 68 & $\begin{array}{l}\text { CP } 42 \\
\text { AP } 17 \\
\text { BC } 32\end{array}$ & $\begin{array}{l}\text { CP 11; } \\
\text { BC } 9\end{array}$ & $\begin{array}{l}\text { CP 42; } \\
\text { AP 17; } \\
\text { BC } 34\end{array}$ & $\begin{array}{l}\text { CP 16; } \\
\text { MBC 13; } \\
\text { LBC } 5\end{array}$ \\
\hline $\begin{array}{l}\text { Unselected/CD34+; } \\
\text { PBMC/BM }\end{array}$ & $\begin{array}{l}\text { CD34; } \\
\text { PBMC }\end{array}$ & $\begin{array}{l}\text { Unselected; } \\
\text { BM }\end{array}$ & $\begin{array}{l}\text { CD34; } \\
\text { PBMC }\end{array}$ & $\begin{array}{l}\text { Unselected; } \\
\text { BM }\end{array}$ & $\begin{array}{l}\text { CD34+; } \\
\text { PBMC }\end{array}$ \\
\hline Platform & Microarray & Microarray & Microarray & Microarray & Microarray \\
\hline N. of genes & 20 & $3000+$ & 114 & 6 & $\begin{array}{l}431 \text { Upregulated } \\
\text { LBC } 522 \text { downregulated }\end{array}$ \\
\hline Comments & $\begin{array}{l}\text { Identifies early ( } \leq 3 \text { years) } \\
\text { vs. late ( } \geq 7 \text { years) BC } \\
\text { transformation. } \\
\text { Low CD7 \& high PR-3 } \\
\text { predicts higher OS. }\end{array}$ & $\begin{array}{l}\text { Identifies TKI-R } \\
\text { in CP (had BC-like } \\
\text { signature) }\end{array}$ & $\begin{array}{l}\text { Genes that } \\
\text { distinguish } \\
\mathrm{CP} \text { and } \mathrm{BC}\end{array}$ & $\begin{array}{l}\text { Discriminates between } \\
\text { early \& late CP }\end{array}$ & $\begin{array}{l}\text { Identifies a core BC } \\
\text { gene expression } \\
\text { signature common to } \\
\text { MBC and LBC. } \\
\text { PRC-driven } \\
\text { transcriptional } \\
\text { reprogramming is } \\
\text { enriched for poor } \\
\text { prognostic genes in CP } \\
\text { in the CD34+ datasets } \\
\text { of Yong et al. (2006) and } \\
\text { McWeeney et al. (2009). }\end{array}$ \\
\hline
\end{tabular}

CP: chronic phase; AP: accelerated phase; BC: blast crisis; MBC: myeloid blast crisis; LBC: lymphoid blast crisis; PBMC: peripheral blood mononuclear cells; BM: bone marrow; PR-

3: proteinase-3: OS: overall survival; TKI-R: resistance to tyrosine kinase inhibitors; PRC: polycomb repressive complex. 
ciated silencing in BC progression were enriched for downregulated genes identified in the datasets of both McWeeney et al. and Yong et al. ${ }^{10}$ The cross-validation of these three independent datasets suggests important lessons for the development of GE-based risk assessment: (i) the discovery of reproducible GE-based biomarkers is possible when homogeneous $\mathrm{CD} 34^{+}$populations are used; (ii) the processes of TKI resistance and $\mathrm{BC}$ transformation are biologically convergent despite genetic heterogeneity: ${ }^{10}$ and (iii). PRC-regulated processes contribute to silencing of prognostically informative genes.

\section{Contribution of somatic mutations to gene expression signatures}

Recent reviews have described the range and frequency of specific genetic mutations in patients who developed TKI resistance and/or BC. ${ }^{37}$ For many of these genes, there is strong preclinical information indicating that their associated mutations contribute to or are even sufficient to produce TKI resistance or transformation phenotypes (summarized in Table 3). ${ }^{38-48}$ These studies imply that genetic mutations alter GE profiles, and here we review their contributions to GE changes in CML since these changes may represent useful GE-based biomarkers.

For RUNX1 mutations, the Mustjoki group identified an accompanying GE signature in BC samples. ${ }^{49}$ They found that RUNX1 mutations were associated with the upregulation of stemness, B-cell markers, interferon and immune signaling and transcription factors regulating plasmacytoid dendritic cell development.

In analogous work, the overexpression of an $I K Z F 1$ dominant-negative mutant in $\mathrm{CD}_{3} 4^{+}$cells from CP patients increased STAT5 expression, a pathway associated with imatinib resistance, ${ }^{50}$ and enhanced transformation. ${ }^{51} R A G$ expression status was recently assessed in diagnostic samples, given the role of RAG recombination as a mediator of IKZF1 deletions. ${ }^{52}$ Notably, RAG1/2 and DNTT upregulation at diagnosis suggested imminent lymphoid BC transformation within 12 months (8/8 patients), demonstrating that GE signatures can reliably predict transformation.

Despite limited functional interrogation of ASXL1 using CML patient material, insertion sites within ASXL1 promoted BC progression in a CP mouse model subjected to transposition-based mutagenesis. ${ }^{53}$ Transgenic expression of truncated protein ASXL1 $1^{\text {aal-587 }}$ in mice increased HSC self-renewal, and Brd4 occupancy and chromatin accessibility around genes required for stemness, and predisposed mice to myeloid malignancies. ${ }^{54}$ However, the clinical relevance of diagnostic ASXL1 mutations is still unclear because some patients with ASXL1 variants at diagnosis can achieve a MMR after TKI therapy. ${ }^{14}$ Furthermore, ASXL1 mutations frequently disappeared when monitored in the long-term during TKI therapy (personal observation by Dr. Dennis Kim). Meanwhile, direct evidence for contributions of other mutations to CML GE signatures is currently lacking, and we have to infer them from studies in other malignancies (Table 3).

\section{Lessons from clonal hematopoiesis}

Clonal hematopoiesis is the clinical phenomenon by which populations of hematopoietic cells expand and carry a somatic mutation that is at least $2 \%$ of the variant allele fraction. ${ }^{55}$ The common genes comprise DNMT3A, TET2, and ASXL1, and others also found in CML individuals, including $R U N X 1, B C O R L 1$, and TP53. ${ }^{56}$ Individuals with clonal hematopoiesis are at increased risk of developing hematologic malignancies, and it is therefore pertinent to ask whether clonal hematopoiesis-related mutations also confer increased risk of TKI resistance or progression. A study by Kim et al. has highlighted important features of clonal hematopoiesis-related mutations in CML. ${ }^{57}$ Firstly, they may occur in a non-Philadelphia chromosome-positive clone and predate the development of CML, and are unrelated to the CML clone. Secondly, even when a specific mutation occurs in the Philadelphia chromosome-positive clone, it only confers a relative risk of TKI resistance or progression. Indeed, patients with RUNX1 mutations have been documented to achieve MMR (personal observation, Dr. Dennis Kim). Nevertheless, Kim et al. concluded that mutations in genes regulating epigenetic function (TET2, ASXL1 among them) were associated with a higher risk of inferior TKI responses.

There are also strong preclinical data indicating that clonal hematopoiesis-related mutations result in subtle but important changes in GE in HSC. For example, Dnmt3a-deficient HSC show a loss of DNA methylation in regions enriched for self-renewal genes such as Meis1, Evil and HoxA9. ${ }^{58}$ In Tet2-deficient mice, the loss of DNA demethylation is accompanied by an expansion of the stem and progenitor cell compartments, and eventual myeloproliferation. ${ }^{45}$ In ASXL1-deficient mice, an increase in self-renewal capacity of stem cells is observed, through the loss of PRC1-mediated gene repression. ${ }^{59}$ Another interesting aspect of hematopoietic stem and progenitor cells harboring inactivating mutations of DNMT3A and TET2 is that they both led to increased cytokine production in peripheral myeloid cells, including interleukin- 6 and interleukin-1 $\beta .^{60,61}$ Furthermore, mutations associated with clonal hematopoiesis are frequently found in monocytes, granulocytes, and natural killer cells compared to B or T cells, suggesting that their effects may also be manifest in multiple differentiated cell types within the hematopoietic compartment. ${ }^{62}$ Together, these observations are relevant to the search for prognostic GE signatures in CML for the following reasons: (i) increased inflammation and cytokine production is associated with LSC persistence, ${ }^{32}$ and disease progression; ${ }^{10,63}$ (ii) prognostic GE changes may be found in both $\mathrm{CD}_{3} 4^{+}$and $\mathrm{CD}^{-}$fractions of peripheral blood or bone marrow mononuclear cells; and (iii) changes in natural killer cell function and number may predict treatment-free remissions, and presumably contain informative natural killer cell GE signatures. ${ }^{64-66}$

\section{Epigenetic contributions to gene expression signatures}

\section{Polycomb repressive complex-associated gene expression changes}

Among the most well studied epigenetic complexes in $\mathrm{CML}$ are the polycomb group (PcG) proteins. ${ }^{67}$ The polycomb group proteins assemble into two complexes, PRC2 and PRC1, which modify histones through repressive H3K27 trimethylation (H3K27me3) and H2AK119 
monoubiquitination (H2AK119ub1), respectively, and in general repress gene expression. ${ }^{6}$

From a mechanistic standpoint, the most commonly occurring mutations in CML appear to converge in their ability to interact with and function in conjunction with the polycomb group proteins. ASXL1 functions in transcriptional repression through its interaction with PRC2 and BAP1 ${ }^{69}$ BCORL1 is a transcriptional co-repressor that interacts with PCGF1, a core complex of the PRC1.1 complex. $^{70}$ The RUNX1-CBF- $\beta$ heterodimer mediates transcription by binding to RUNX sites, but also represses transcription by interacting and recruiting BMI1 of the PRC1 complex to target sites. ${ }^{71}$ IKZF1 regulates transcription by interacting with repressive epigenetic complexes such as HDAC1, HDAC2, CHD3, CHD4, and SWI/SNF complex, and also recruits PRC2 to target gene loci in $\mathrm{T}$ cells. $^{72}$ Thus, while the commonly mutated genes in CML have their own exclusive roles in transcriptional regulation, they also share a striking commonality as modulators of the PRC. Whether mutated variants of RUNX1,
ASXL1, IKZF1, and BCORL1 drive aberrant PRC recruitment and GE in CML remains to be determined.

In this respect, a recent study determined that lymphoid and myeloid BC transcriptomes are highly congruent, and that both undergo PRC-driven epigenetic reprogramming towards a convergent transcriptomic state. ${ }^{10}$ PRC-dependent epigenetic reprogramming was attributed to gain- and loss-of-function mutations in members of the PRC1 and PRC2 complexes, respectively. Of these, ongoing BMI1/PRC1 activity contributes to maintaining the BC transcriptome, while EZH2/PRC2-binding was instructional for DNA hypermethylation-dependent gene repression. Importantly, the integrative model proposed by Ko et al. suggests that enrichment for PRC-dependent GE signatures at diagnosis can predict disease transformation and TKI resistance, as highlighted above. ${ }^{10}$

We also note that dysregulated regulation of PRC has been identified as a key feature of TKI-resistant LSC in CP. EZH2 expression was higher in CML LSC than in normal HSC, and CML LSC have a stronger dependence on

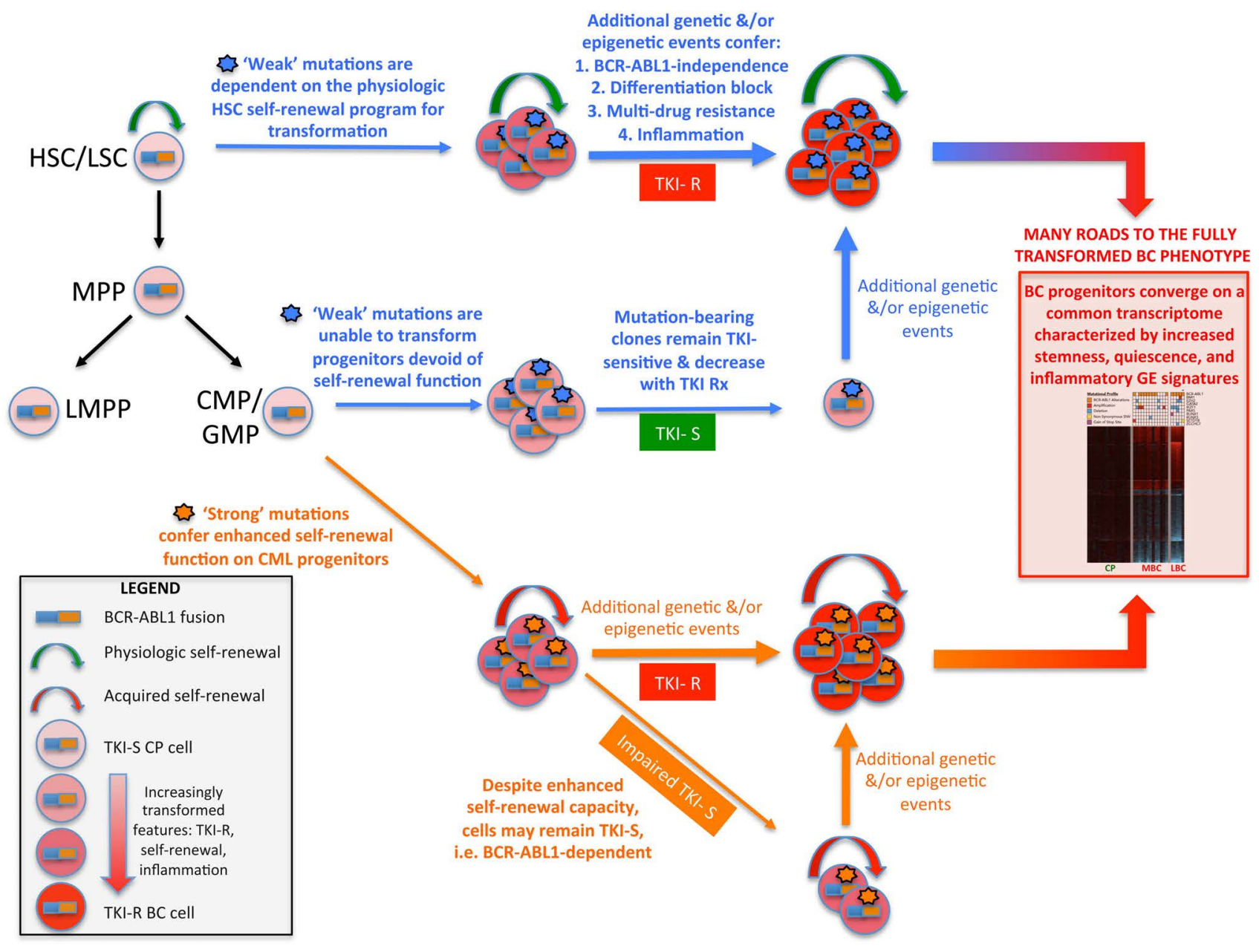

Figure 2. Diagrammatic representation of the 'seed and soil' model of chronic myeloid leukemia. The model proposes that both acquired mutations and the cell state of the mutation-acquiring cell contribute to the process of full transformation to blast crisis (BC). A 'strong' mutation is defined as being able to confer selfrenewal function on a progenitor cell that does not possess inherent self-renewal capacity. A 'weak' mutation is unable to confer self-renewal function and can only transform a cell with native self-renewal ability, i.e., a stem cell. For both strong and weak mutations, it is likely that additional genetic and epigenetic events are necessary to confer the full suite of features required for $\mathrm{BC}$ transformation. The model is also based on the recent finding that BC progenitors which harbor different somatic mutations share a common or core transcriptome enriched for stemness, quiescence, and inflammatory gene expression signatures..$^{20} \mathrm{HSC}$ : hematopoietic stem cell; LSC: leukemia stem cell; MPP: multipotential progenitor; LMPP: lymphoid-primed multipotent progenitor; CMP: common myeloid progenitor; GMP: granulocyte-macrophage progenitor; TKI-S/R: tyrosine kinase inhibitor-sensitive/resistant cells. Rx: treatment; CP: chronic phase; BC: blast crisis; CML: chronic myeloid leukemia; GE: gene expression. 


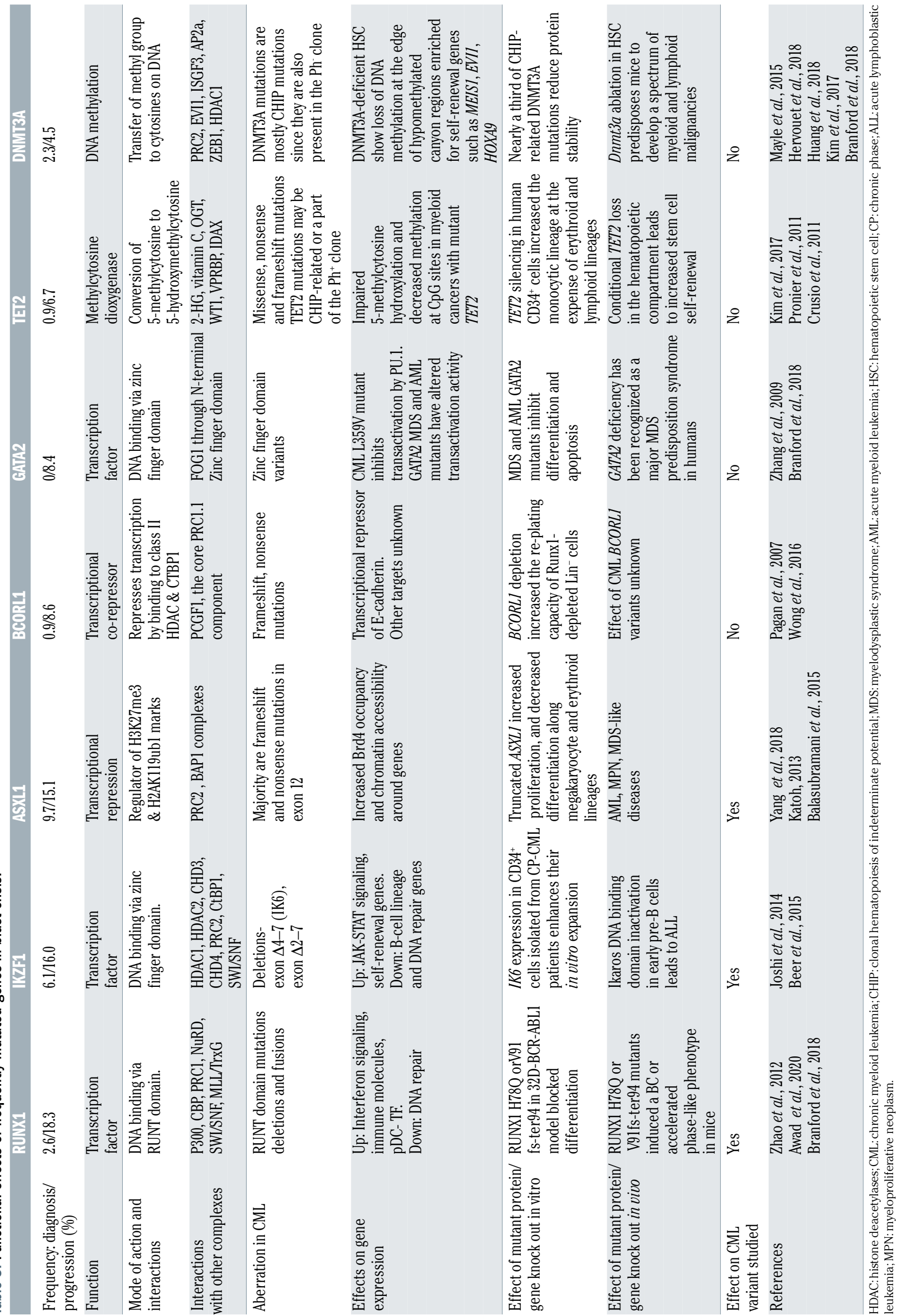


the PRC2-EZH2 axis for survival and TKI resistance. ${ }^{73,74}$ Likewise, higher BMI1 levels at diagnosis correlated with disease progression from $\mathrm{CP}$ to $\mathrm{BC} .{ }^{12}$ while $B M I 1$ overexpression in $\mathrm{CP} \mathrm{CD} 34^{+}$cells increased proliferation and self-renewal, ${ }^{75}$ and transformed B-lymphoid progenitors in vivo. ${ }^{76}$

\section{DNA methylation-associated gene expression changes}

Many studies have examined the role of DNA methylation as a regulator of aberrant GE in CML pathogenesis. In candidate-based approaches, genes involved in cell cycle regulation (P16, P53, PLCD1, PER3, HIC1), differentiation (HOXA4, DLX4, DDIT3, SPI1) proliferation (CDH13, $D A P K 1)$, apoptosis (BIM), Wnt regulation (sFRP1, CBY1), LSC maintenance (MTSS1), and cell signaling (Jun B, SOCS2) were identified as targets of DNA methylation. ${ }^{6,77}$

Recent unbiased genome-wide methylome analyses have solidified the concept of aberrant DNA methylation as a driver of resistance and transformation. The number of differentially methylated regions in $\mathrm{CP}$ increased from $\sim 600$ to $~ 6,500 \mathrm{CpG}$ sites in BC. ${ }^{78} \mathrm{BC}$ was associated with heightened DNA hypermethylation, and to a lesser extent hypomethylation, around promoters of genes involved in stem cell fate, differentiation and leukemia-related functions. ${ }^{10}$ Mechanistically, differential DNA methylation patterns in CML have been attributed to underlying DNMT3A/TET2 mutations, PRC2-dependent epigenetic re-programming, and cytosolic sequestration of Tet 2 by BCR-ABL1. ${ }^{79}$ Notably, the physiological targeting of DNA hypermethylation using 5-aza-2'-deoxycytidine ameliorated disease phenotypes in a mouse model of $\mathrm{CP}$ disease ${ }^{80}$ while low-dose decitabine displayed clinical activity in patients refractory to imatinib, ${ }^{81}$ suggesting DNA methylation does indeed contribute to TKI resistance.

Based on the biological insights gleaned so far, it is possible that progression-related DNA methylation signatures may already be evident at diagnosis, particularly in patients presenting with advanced CP. ${ }^{10}$ The DNA methylation status of specific target genes might therefore be useful in the timely identification of such patients for more aggressive therapies. Furthermore, given that DNA methylation is a relatively stable epigenetic and biochemical mark, there are practical advantages to developing DNA methylation-based biomarkers rather than transcript-based readouts, especially for the development of robust clinical-grade tests (Figure 3).

\begin{tabular}{|c|c|c|}
\hline STAGE 0 & STAGE I & STAGE II \\
\hline $\begin{array}{c}\text { Discovery Phase } \\
\text { Identification of genes associated } \\
\text { with clinically meaningful group }\end{array}$ & $\begin{array}{l}\text { Analytical Validity } \\
\text { Accurately \& reliably measure } \\
\text { GE-related phenotype }\end{array}$ & $\begin{array}{l}\text { Clinical Validity } \\
\text { Differentiates biologically or } \\
\text { clinically meaningful groups }\end{array}$ \\
\hline $\begin{array}{l}\text { Unbiased vs candidate approach } \\
\text { - Use of well-annotated samples } \\
\text { using standard response criteria } \\
\text { - Consider stability of material to } \\
\text { be assayed, e.g. RNA, DNA } \\
\text { - Consider performance } \\
\text { characteristics of discovery } \\
\text { platform: biased or unbiased } \\
\text { - Consider technical \& statistical } \\
\text { robustness of discovery pipeline }\end{array}$ & $\begin{array}{l}\text { Define test methodology } \\
\text { - Assay platform: } \\
\text { - mRNA-based (RT-PCR, ISH, } \\
\text { LCM, microarray, nanostring) } \\
\text { - Protein-based (conventional } \\
\text { and multiplex IHC, FC) } \\
\text { - DNA-based (e.g. DNA } \\
\text { methylation) } \\
\text { - High-dimensional single-cell } \\
\text { technologies (mass cytometry }\end{array}$ & $\begin{array}{l}\text { Identify biologic and/or clinical } \\
\text { outcome of interest } \\
\text { - Upfront response to first-line } \\
\text { TKI per NCCN/ELN criteria } \\
\text { - Response to } 2 \text { nd and } \\
\text { subsequent TKI } \\
\text { - BC transformation rate } \\
\text { - EFS, DFS, PFS, OS } \\
\text { - TFR rates }\end{array}$ \\
\hline
\end{tabular}

Gene set curation

- Preferably informed by biological understanding, e.g. stemnessrelated, proliferation, and disease progression

- Identify minimum gene set that distinguishes biologically or clinically meaningful groups

- Level of GE-related variable should robustly distinguish between groups

- Consider and/or assess if gene products (mRNA, protein) or factors regulating GE (e.g. DNA methylation of gene promoter) can be reliably assayed using standard clinical platforms, e.g. IHC, FC, RT-PCR, bisulfite PCR

- Readout (e.g. mRNA) should be stable, and detectable in tissues that are routinely available in standard of care settings technologies (mass cytometry, scRNA/ATAC-seq)

- Assess test sensitivity, specificity, reliability, and robustness for measurement of gene set from DEV Stage 0

- Inclusion of appropriate controls, e.g. housekeeping genes

\section{Determine tissue \&} cell type to test

- Tissue should represent that which is routinely collected as part of clinical care, e.g BM at diagnosis, PBMC at diagnosis and follow ups

- BM:

- MNC:

- CD34+-selected/unselected

- FFPE

- PBMC:

- CD34+-selected/unselected

- Other cell types: NK, MDSCS
Identification of cohort for testing

- Availability and completeness of clinical data e.g. risk factors, therapeutic history

- Adequate description of study population:

- Inclusion/exclusion criteria

- Sample size

- Demographics

- Detailed annotation of clinical risk factors, therapeutic history

Data analysis

Blinded testing and result interpretation

- Identify potential biases in study \& impact on study

- Provide estimates of sensitivity and specificity with $95 \% \mathrm{C}$

- Provide estimates of positive and negative prodictive values

Figure 3. Stages of development of gene expression-based biomarkers. In chronic myeloid leukemia (CML), the development of gene expression-based biomarkers can be divided into three stages following an initial discovery phase. These stages will each determine the analytical validity, clinical validity, and clinical utility of the tests in question. Examples of CML-specific issues or questions that are pertinent to each stage are outlned in boxes under each stage. GE: gene expression; IHC: immunohistochemistry; FC: flow cytometry; RT-PCR: reverse transcriptase polymerase chain reaction; ISH: in-situ hybridization; LCM: laser capture microdissection; scRNA-seq: single-cell RNA sequencing; ATAC-seq: assay for transposase-accessible chromatin sequencing; BM: bone marrow; PBMC: peripheral blood mononuclear cell; MNC: mononuclear cells; FFPE: formalin-fixed paraffin-embedded tissues; PB: peripheral blood; NK: natural killer cells; MDSC: myeloid-derived suppressor cells; TKI: tyrosine kinase inhibitor; NCCN: National Comprehensive Cancer Network; ELN: European LeukemiaNet; BC: blast crisis; EFS: event-free survival; DFS: diseasefree survival; PFS: progression-free survival; OS: overall survival, TFR: treatment-free remission; 95\% Cl: 95\% confidence interval; DMR: deep molecular response.. 
Gene expression profiles and mutations: 'seed and soll' revisited

As described above, it will be important to develop CML models that integrate the interaction between genetic and epigenetic factors in driving drug resistance and disease transformation. In this respect, the effects of specific mutations may be cell-context dependent, with differential effects on GE and function depending on the cell type being examined. This is particularly the case for mutations affecting transcription factors, for which cell states, and their attendant chromatin accessibility profile, determine whether the mutated transcription factor has access to its target genes.

To integrate contributions from both the above features, we propose a model in which the cell of origin, with its attendant epigenetic and transcriptional program, determines the ability of specific mutations to contribute to biological and clinical outcomes (Figure 2). This model is a derivative of the 'seed and soil' hypothesis of cancer initiation. ${ }^{82}$ The model will be useful for hypothesis testing, and likely explains an important feature of BCRABL1 itself. It has been shown in murine models that only when expressed in HSC, but not more committed progenitors, can BCR-ABL1 induce a myeloproliferative disorder. This is likely because BCR-ABL1 is incapable of conferring self-renewal capacity upon committed progenitors, indicating that CML cells rely on BCR-ABL1-independent mechanisms for stemness programs. These findings are in contrast to those for other leukemia fusion genes (e.g., MLL-ENL, MLL-AF9, MOZ-TIF2) which are capable of conferring self-renewal and transform progenitor cells. ${ }^{83}$ Relatedly, the model may also explain a naturally occurring phenomenon whereby normal individuals found to carry the $B C R-A B L 1$ fusion in their peripheral blood mononuclear cells apparently never develop CML ${ }^{84}$ Here, the model would posit that the BCR-ABL1 fusion is occurring in a long-lived progenitor without selfrenewal function.

Analogous to the situation regarding cancer initiation by leukemia fusion genes, mutations devoid of selfrenewal function may only confer an increased risk of $B C$ transformation when they occur in a target cell that already possesses physiological self-renewal function. According to this model, mutations in RUNX1 that are sufficient to induce BC-like disease in mice (Table 3) may be deemed a 'strong' biological seed that can transform many cell types within the hematopoietic hierarchy. Such mutations would be expected to induce disease progression in the majority of patients who harbor such mutations, which is indeed the case. ${ }^{37}$ However, a minority of $\mathrm{CP}$ patients with RUNX1 mutations continue to enjoy sustained deep maolecular responses ${ }^{57}$ suggesting the existence of other important factors that modulate RUNX1 function. Along the same lines, ASXL1 was recently identified as the most frequently mutated gene at diagnosis in nine patients, the majority $(\mathrm{n}=6)$ of whom eventually developed $B C$, while a minority $(n=2)$ achieved a MMR. ${ }^{14}$

In contrast to the above examples, the prognostic impact of 'weak' seeds is much less clear. In a study by Kim et al., at least four different patterns were observed for TET2 mutations. ${ }^{57}$ One pattern is seen in patients with TKI resistance when both TET2 and ABL1 variant allele frequencies increased following TKI therapy, while another is seen when the TET2 variant allele frequency reduces after TKI treatment in patients with disease progression. In other cases, TET2 mutations were also detected within Philadelphia chromosome-negative cells, and here, patients showed complex outcomes following TKI therapy, with some achieving MMR and others showing TKI resistance. These observations suggest that the effect of TET2 mutations are highly contextual.

\section{Challenges ahead but room for optimism}

As described above, the discovery of a limited and tractable set of genes that is prognostic across a majority of CML patients has been challenging for clinical, biological, and technical reasons. Nevertheless, there is room for optimism. In the setting of breast cancer, GE panels comprising 21 genes that encompass various aspects of breast cancer biology have been found to be predictive of therapeutic response, and minimized the use of additional therapy without compromising survival. ${ }^{85}$ Among liquid tumors, a recent study in acute myeloid leukemia demonstrated that a parsimonious 17-gene GE score, derived from a larger set of stemness-conferring genes, predicts resistance to initial therapy. ${ }^{86}$ Interestingly, this score was independent of cytogenetic and mutational risk factors, and suggests that biological factors (e.g., stemness) transcend traditional genetics-based groupings. ${ }^{87}$

Encouragingly in CML, two recent reports suggest that it is possible, using peripheral blood samples taken at diagnosis or 3 months after diagnosis, to predict deep molecular responses and also sustained treatment-free remissions. In the first study, the Adelaide group showed that the rate of decline of $B C R-A B L 1$ transcripts during first-line TKI therapy (calculated from baseline and 3month $B C R-A B L 1$ transcript levels) predicts success of treatment-free remission. ${ }^{88}$ The time taken for BCR-ABL1 transcripts to halve was the strongest independent predictor of sustained treatment-free remission: $80 \%$ in patients with a halving time of $<9.35$ days versus $4 \%$ if the halving time was $>21.85$ days $(\mathrm{P}<0.001)$. In a separate study, Radich et al. reported that GE signatures from peripheral blood taken prior to TKI initiation can distinguish individuals who will achieve a deep molecular response (MR4.5) at 5 years from those who will have suboptimal responses. ${ }^{89}$ Thus, biological information encoded in GE data can predict very long-term clinical outcomes in CML, and it is therefore conceivable that GE-based data will be able to identify patients in whom TKI therapy can be safely discontinued. More importantly, these early reports suggest that despite the likely existence of diverse resistance mechanisms within the study populations, final common paths, readout either as dynamic measures of $B C R-A B L 1$ transcript levels, or peripheral blood GE signatures are indeed discoverable.

\section{Stages in developing gene expression-based risk assessment}

The stages of developing GE-based tests has been outlined in recent reviews and consensus statements, and comprise at least three phases that assess: analytical validity (reliably measuring the genotype of interest), clinical validity (ability to segregate patients into biologi- 
cally and clinically important subsets), and clinical utility (ability to improve clinical decision making). ${ }^{90,91}$ In this section, we summarize the pertinent stages and highlight issues of particular relevance to GE-based biomarker development in CML (Figure 3).

Stage 0 is the discovery stage, which is where the field is currently. Here, we highlight three important components, which include the use of technical approaches for unbiased discovery, the simultaneous interrogation of leukemic and non-leukemic clones from the same sample (since both have been shown to be prognostic), and the use of robust statistical and computational pipelines to discover minimal prognostic genes sets. The advent of single-cell-based technologies and their application to well-annotated cohorts will facilitate this step.

In stage I, the minimal gene set has to be converted into a clinical test that accurately and reproducibly measures the GE phenotype. The test platform needs to be robust, as well as sensitive, specific and reliable. The assay should be developed for tissues that are collected as part of routine clinical care. Ideally, any additional processing of material beyond what is routine should be minimized, e.g., CD34 ${ }^{+}$selection, and should utilize standard procedures available in clinical laboratories, such as flow cytometry and bone marrow immunohistochemistry. An example would be detecting GE signatures of interest by a panel of antibodies for use in flow cytometry or immunohistochemistry applications. It is preferable that the samples used for analytical validation are from wellcharacterized patients representative of 'real-world' settings and, ideally, validated in at least one independent cohort. Sample size and power calculations should be determined prior to starting the study, and analytic sensitivity and specificity for the test should be available at the end of the study. At the end of stage I, a locked-down test should be evaluated in stage II, that of clinical validation.

In stage II, the locked-down test will be evaluated for its ability to differentiate between clinically meaningful outcomes in modern CML practice. The samples to be tested should be obtained from well-annotated cohorts representative of the broader population, and the test conducted on tissues in a blinded manner with respect to testing and result reporting. Ideal populations include patients who have been treated uniformly in clinical trials. At the end of this stage, the ability of the test to predict clinical outcome should be available as a test score, with clearly defined positive and negative predictive values.

The final stage, stage III, will be the determination of clinical utility. This stage would entail the use of the GEbased test to improve clinical decision-making, and would require the study to demonstrate that meaningful outcomes are improved when the test is used compared to when the test is not used. Besides clinical outcomes such as improved progression-free survival and overall survival, additional measures such as cost-effectiveness, avoidance of toxicities, quality of life and psychological parameters should also be assessed. Such studies may also incorporate the contribution of pharmacological factors (e.g., drug metabolism and side effects, patient com- pliance) to overall outcomes. Given the relative rarity of CML, it is envisaged that this will be a multicenter international study.

\section{Conclusion}

Genetic and epigenetic events contribute to the emergence of BCR-ABL1-independent clones that result in clinical TKI resistance and, if unopposed, BC transformation. Long-term TKI responses, including successful TKI stoppage, can be predicted by slower declines in $B C R$ $A B L 1$ transcript levels during first-line TKI therapy, ${ }^{88}$ suggesting that genetic and epigenetic factors contributing to TKI resistance are present at diagnosis. Recent studies describe a convergent GE signature common to the majority of $\mathrm{BC}$ progenitors. ${ }^{10}$ Elements of this common or core transcriptome can be detected in $\mathrm{CD} 34^{+}$cells from CP patients at risk of TKI resistance or early transformation, ${ }^{11,13}$ and specific mutations have been shown to contribute additional nuances to the core transcriptome. ${ }^{49}$ These observations are consistent with a 'seed and soil' model that may be helpful for hypothesis generation (Figure 2). Emerging technologies, particularly multimodal single-cell-based approaches, will facilitate the discovery of genetic and epigenetic biomarkers at presentation. This initial discovery phase has to be followed by the translation of GE-based information into validated analytical tests, and subsequently, the determination of clinical validity and utility. This process will be a multi-year, multi-institution international effort akin to that for the development of a genetic-based risk assessment. ${ }^{85,90,91}$ The integration of both gene mutation- and gene expression-based biomarkers into the care of CML patients will be an important step to achieving the ultimate goal of CML research: the cure of the majority of our patients.

\section{Disclosures}

$D K$ is a member of the advisory boards of Novartis, Pfizer, Paladin, and has received honoraria from Novartis, Pfizer and Paladin, as well as research funding from Novartis, BristolMyers Squibb, Pfizer, and Paladin. TH is a member of a Novartis advisory board and receives research support from Novartis and Bristol-Myers Squibb. SB is a member of the advisory boards of Qiagen, Novartis, and Cepheid and has received honoraria from Qiagen, Novartis, Bristol-Myers Squibb, and Cepheid, as well as research support from Novartis and Cepheid.

\section{Contributions}

$V K$ and STO conceived the topic for review, and wrote the first draft of the manuscript. DDHK, TPH, and SB contributed by the addition of new sections and critical discussions throughout the writing of the review.

\section{Funding}

VK and STO are supported by the National Medical Research Council Singapore (MOH-CSASI18may-0002, MOH-CIRG2Onov-0003, NMRC/CIRG/1468/2017). 


\section{References}

1. Rowley JD. Letter: A new consistent chromosomal abnormality in chronic myelogenous leukaemia identified by quinacrine fluorescence and Giemsa staining. Nature. 1973;243(5405):290-293.

2. Perrotti D, Jamieson C, Goldman J, Skorski T. Chronic myeloid leukemia: mechanisms of blastic transformation. J Clin Invest. 2010;120(7):2254-2264.

3. Druker BJ. Translation of the Philadelphia chromosome into therapy for CML. Blood. 2008;112(13):4808-4817.

4. Holyoake TL, Vetrie D. The chronic myeloid leukemia stem cell: stemming the tide of persistence. Blood. 2017;129(12): 1595-1606

5. Hochhaus A, Baccarani M, Silver RT, et al. European LeukemiaNet 2020 recommendations for treating chronic myeloid leukemia. Leukemia. 2020;34(4):966-984.

6. Mahon FX, Rea D, Guilhot J, et al. Discontinuation of imatinib in patients with chronic myeloid leukaemia who have maintained complete molecular remission for at least 2 years: the prospective, multicentre Stop Imatinib (STIM) trial. Lancet Oncol. 2010;11(11):1029-1035.

7. Ross DM, Hughes TP. Treatment-free remission in patients with chronic myeloid leukaemia. Nat Rev Clin Oncol. 2020;17 (8):493-503.

8. Branford S, Fletcher L, Cross NC, et al. Desirable performance characteristics for BCR-ABL measurement on an international reporting scale to allow consistent interpretation of individual patient response and comparison of response rates between clinical trials. Blood. 2008;112(8):3330-3338.

9. Malhotra H, Radich J, Garcia-Gonzalez P. Meeting the needs of CML patients in resource-poor countries. Hematology Am Soc Hematol Educ Program. 2019;2019 (1):433-442.

10. Ko TK, Javed A, Lee KL, et al. An integrative model of pathway convergence in genetically heterogeneous blast crisis chronic myeloid leukemia. Blood. 2020;135 (26):2337-2353.

11. McWeeney SK, Pemberton LC, Loriaux $\mathrm{MM}$, et al. A gene expression signature of CD34+ cells to predict major cytogenetic response in chronic-phase chronic myeloid leukemia patients treated with imatinib. Blood. 2010;115(2):315-325.

12. Mohty M, Yong AS, Szydlo RM, Apperley JF, Melo JV. The polycomb group BMI1 gene is a molecular marker for predicting prognosis of chronic myeloid leukemia. Blood. 2007;110(1):380-383.

13. Yong AS, Szydlo RM, Goldman JM, Apperley JF, Melo JV. Molecular profiling of CD34+ cells identifies low expression of CD7, along with high expression of proteinase 3 or elastase, as predictors of longer survival in patients with CML. Blood. 2006;107(1):205-212

14. Branford S, Wang P, Yeung DT, et al. Integrative genomic analysis reveals cancerassociated mutations at diagnosis of $\mathrm{CML}$ in patients with high-risk disease. Blood. 2018;132(9):948-961.

15. Malone ER, Oliva M, Sabatini PJB, Stockley TL, Siu LL. Molecular profiling for precision cancer therapies. Genome Med. 2020;12 (1):8.

16. Kaneta Y, Kagami Y, Katagiri T, et al. Prediction of sensitivity to STI571 among chronic myeloid leukemia patients by genome-wide cDNA microarray analysis. Ipn J Cancer Res. 2002;93(8):849-856.

17. McLean LA, Gathmann I, Capdeville R, Polymeropoulos $\mathrm{MH}$, Dressman $\mathrm{M}$ Pharmacogenomic analysis of cytogenetic response in chronic myeloid leukemia patients treated with imatinib. Clin Cancer Res. 2004;10(1 Pt 1):155-165.

18. de Lavallade H, Finetti P, Carbuccia N, et al. A gene expression signature of primary resistance to imatinib in chronic myeloid leukemia. Leuk Res. 2010;34(2):254-257.

19. Zhang WW, Cortes JE, Yao H, et al. Predictors of primary imatinib resistance in chronic myelogenous leukemia are distinct from those in secondary imatinib resistance. J Clin Oncol. 2009;27(22):3642-3649.

20. Kok CH, Yeung DT, Lu L, et al. Gene expression signature that predicts early molecular response failure in chronic-phase CML patients on frontline imatinib. Blood Adv. 2019;3(10):1610-1621

21. Marin D, Ibrahim AR, Lucas $C$, et al. Assessment of BCR-ABL1 transcript levels at 3 months is the only requirement for predicting outcome for patients with chronic myeloid leukemia treated with tyrosine kinase inhibitors. J Clin Oncol. 2012;30(3): 232-238.

22. Hughes TP, Saglio G, Kantarjian HM, et al Early molecular response predicts out comes in patients with chronic myeloid leukemia in chronic phase treated with frontline nilotinib or imatinib. Blood. 2014;123(9):1353-1360.

23. Frank O, Brors B, Fabarius A, et al. Gene expression signature of primary imatinib resistant chronic myeloid leukemia patients. Leukemia. 2006;20(8):1400-1407.

24. Villuendas R, Steegmann JL, Pollan M, et al Identification of genes involved in imatinib resistance in CML: a gene-expression profiling approach. Leukemia. 2006;20(6) 1047-1054.

25. Radich JP, Dai H, Mao M, et al. Gene expression changes associated with progression and response in chronic myeloid leukemia. Proc Natl Acad Sci U S A. 2006;103(8):2794-2799.

26. Lim S, Saw TY, Zhang M, et al. Targeting of the MNK-eIF4E axis in blast crisis chronic myeloid leukemia inhibits leukemia stem cell function. Proc Natl Acad Sci U S A. 2013;110(25):E2298-2307.

27. Crossman LC, Mori M, Hsieh YC, et al. In chronic myeloid leukemia white cells from cytogenetic responders and non-responders to imatinib have very similar gene expression signatures. Haematologica. 2005;90(4): 459-464

28. Burguillo FJ, Martin J, Barrera I, Bardsley WG. Meta-analysis of microarray data: the case of imatinib resistance in chronic myelogenous leukemia. Comput Biol Chem. 2010;34(3):184-192.

29. Jamieson CH, Ailles LE, Dylla SJ, et al. Granulocyte-macrophage progenitors as candidate leukemic stem cells in blast-crisis CML. N Engl J Med. 2004;351(7):657-667.

30. Lim B, Lin Y, Navin N. Advancing cancer research and medicine with single-cell genomics. Cancer Cell. 2020;37(4):456-470.

31. Warfvinge R, Geironson L, Sommarin $\mathrm{MNE}$, et al. Single-cell molecular analysis defines therapy response and immunophenotype of stem cell subpopulations in CML. Blood. 2017;129(17):2384-2394.

32. Giustacchini A, Thongjuea S, Barkas N, et al. Single-cell transcriptomics uncovers distinct molecular signatures of stem cells in chronic myeloid leukemia. Nat Med.
2017;23(6):692-702

33. Inselmann S, Wang Y, Saussele S, et al. Development, function, and clinical significance of plasmacytoid dendritic cells in chronic myeloid leukemia. Cancer Res. 2018;78(21):6223-6234.

34. Sinnakannu JR, Lee KL, Cheng $S$, et al. SRSF1 mediates cytokine-induced impaired imatinib sensitivity in chronic myeloid leukemia. Leukemia. 2020;34(7):1787-1798.

35. Oehler VG, Yeung KY, Choi YE, et al. The derivation of diagnostic markers of chronic myeloid leukemia progression from microarray data. Blood. 2009;114(15):32923298

36. Zheng C, Li L, Haak M, et al. Gene expression profiling of CD34+ cells identifies a molecular signature of chronic myeloid leukemia blast crisis. Leukemia. 2006;20(6): 1028-1034.

37. Branford S, Kim DDH, Apperley JF, et al. Laying the foundation for genomicallybased risk assessment in chronic myeloid leukemia. Leukemia. 2019;33(8):1835-1850.

38. Zhao LJ, Wang YY, Li G, et al. Functional features of RUNX1 mutants in acute transformation of chronic myeloid leukemia and their contribution to inducing murine fullblown leukemia. Blood. 2012;119(12):28732882

39. Joshi I, Yoshida T, Jena N, et al. Loss of Ikaros DNA-binding function confers integrin-dependent survival on pre-B cells and progression to acute lymphoblastic leukemia. Nat Immunol. 2014;15(3):294 304.

40. Balasubramani A, Larjo A, Bassein JA, et al. Cancer-associated ASXL1 mutations may act as gain-of-function mutations of the ASXL1-BAP1 complex. Nat Commun. 2015;6:7307.

41. Pagan JK, Arnold J, Hanchard KJ, et al. A novel corepressor, BCoR-L1, represses transcription through an interaction with $\mathrm{CtBP}$. J Biol Chem. 2007;282(20):15248-15257.

42. Kazenwadel J, Secker GA, Liu YJ, et al. Loss-of-function germline GATA2 mutations in patients with MDS/AML or MonoMAC syndrome and primary lymphedema reveal a key role for GATA2 in the lymphatic vasculature. Blood 2012;119(5):1283-1291.

43. Zhang SJ, Shi JY, Li JY. GATA-2 L359V mutation is exclusively associated with CML progression but not other hematological malignancies and GATA-2 P250A is a novel single nucleotide polymorphism. Leuk Res. 2009;33(8):1141-1143.

44. Pronier E, Almire C, Mokrani $\mathrm{H}$, et al Inhibition of TET2-mediated conversion of 5 -methylcytosine to 5-hydroxymethylcytosine disturbs erythroid and granulomonocytic differentiation of human hematopoietic progenitors. Blood. 2011 118(9):2551-2555

45. Moran-Crusio K, Reavie L, Shih A, et al. Tet2 loss leads to increased hematopoietic stem cell self-renewal and myeloid transformation. Cancer Cell. 2011;20(1):11-24.

46. Mayle A, Yang L, Rodriguez B, et al. Dnmt3a loss predisposes murine hematopoietic stem cells to malignant transformation. Blood. 2015;125(4):629638.

47. Hervouet E, Peixoto P, Delage-Mourroux R Boyer-Guittaut M, Cartron PF. Specific or not specific recruitment of DNMTs for DNA methylation, an epigenetic dilemma. Clin Epigenetics. 2018;10:17.

48. Huang Y-H, Tovy A, Sundaramurthy V, et al. Nearly a third of clonal hematopoiesis- 
associated DNMT3A mutations reduce protein stability and may be associated with poorer prognosis. Blood. 2018;132 (Suppl 1):1315

49. Adnan Awad S, Dufva O, Ianevski A, et al. RUNX1 mutations in blast-phase chronic myeloid leukemia associate with distinct phenotypes, transcriptional profiles, and drug responses. Leukemia. 2021;35(4): 1087-1099.

50. Warsch W, Kollmann K, Eckelhart E, et al. High STAT5 levels mediate imatinib resistance and indicate disease progression in chronic myeloid leukemia. Blood. 2011;117 (12):3409-3420.

51. Beer PA, Knapp DJ, Miller PH, et al. Disruption of IKAROS activity in primitive chronic-phase CML cells mimics myeloid disease progression. Blood. 2015;125(3): 504-515.

52. Thomson DW, Shahrin NH, Wang PPS, et al. Aberrant RAG-mediated recombination contributes to multiple structural rearrangements in lymphoid blast crisis of chronic myeloid leukemia. Leukemia. 2020;34(8): 2051-2063.

53. Giotopoulos G, van der Weyden L, Osaki $\mathrm{H}$, et al. A novel mouse model identifies cooperating mutations and therapeutic targets critical for chronic myeloid leukemia progression. J Exp Med. 2015;212(10):15511569.

54. Yang H, Kurtenbach S, Guo Y, et al. Gain of function of ASXL1 truncating protein in the pathogenesis of myeloid malignancies. Blood. 2018;131(3):328-341.

55. Jaiswal S, Ebert BL. Clonal hematopoiesis in human aging and disease. Science. 2019; 366(6465):eaan4673.

56. Schmidt M, Rinke J, Schafer V, et al. Molecular-defined clonal evolution in patients with chronic myeloid leukemia independent of the BCR-ABL status. Leukemia. 2014;28(12):2292-2299.

57. Kim T, Tyndel MS, Kim HJ, et al. Spectrum of somatic mutation dynamics in chronic myeloid leukemia following tyrosine kinase inhibitor therapy. Blood. 2017;129 (1):38-47.

58. Jeong $M$, Sun D, Luo $M$, et al. Large conserved domains of low DNA methylation maintained by Dnmt3a. Nat Genet. 2014;46(1):17-23.

59. Abdel-Wahab O, Gao J, Adli M, et al. Deletion of Asxl1 results in myelodysplasia and severe developmental defects in vivo. J Exp Med. 2013;210(12):2641-2659.

60. Sano S, Oshima K, Wang Y, et al. CRISPRmediated gene editing to assess the roles of Tet2 and Dnmt3a in clonal hematopoiesis and cardiovascular disease. Circ Res. 2018;123(3):335-341

61. Sano S, Oshima K, Wang Y, et al. Tet2mediated clonal hematopoiesis accelerates heart failure through a mechanism involving the IL-1beta/NLRP3 inflammasome. J Am Coll Cardiol. 2018;71(8):875-886.

62. Arends CM, Galan-Sousa J, Hoyer K, et al. Hematopoietic lineage distribution and evolutionary dynamics of clonal hematopoiesis. Leukemia. 2018;32(9):19081919.

63. Welner RS, Amabile G, Bararia D, et al Treatment of chronic myelogenous leukemia by blocking cytokine alterations found in normal stem and progenitor cells. Cancer Cell. 2015;27(5):671-681.

64. Hughes A, Yong ASM. Immune effector recovery in chronic myeloid leukemia and treatment-free remission. Front Immunol. 2017;8:469.

65. Hughes A, Clarson J, Tang C, et al. CML patients with deep molecular responses to TKI have restored immune effectors and decreased PD-1 and immune suppressors. Blood. 2017;129(9):1166-1176.

66. Yong AS, Keyvanfar K, Hensel N, et al. Primitive quiescent CD34+ cells in chronic myeloid leukemia are targeted by in vitro expanded natural killer cells, which are functionally enhanced by bortezomib. Blood. 2009;113(4):875-882.

67. Koschmieder S, Vetrie D. Epigenetic dysregulation in chronic myeloid leukaemia: a myriad of mechanisms and therapeutic options. Semin Cancer Biol. 2018;51:180 197.

68. Sparmann A, van Lohuizen M. Polycomb silencers control cell fate, development and cancer. Nat Rev Cancer. 2006;6(11):846-856.

69. Katoh M. Functional and cancer genomics of ASXL family members. Br J Cancer 2013;109(2):299-306.

70. Wong SJ, Gearhart MD, Taylor $\mathrm{AB}$, et al. KDM2B recruitment of the polycomb group complex, PRC1.1, requires cooperation between PCGF1 and BCORL1. Structure. 2016;24(10):1795-1801.

71. Yu M, Mazor T, Huang $\mathrm{H}$, et al. Direct recruitment of polycomb repressive complex 1 to chromatin by core binding transcription factors. Mol Cell. 2012:45(3):330343.

72. Oravecz A, Apostolov A, Polak K, et al. Ikaros mediates gene silencing in $\mathrm{T}$ cells through Polycomb repressive complex 2 . Nat Commun. 2015;6:8823.

73. Xie H, Peng C, Huang J, et al. Chronic myelogenous leukemia-initiating cells require polycomb group protein EZH2 Cancer Discov. 2016:6(11):1237-1247.

74. Scott MT, Korfi K, Saffrey P, et al. Epigenetic reprogramming sensitizes CMI stem cells to combined EZH2 and tyrosine kinase inhibition. Cancer Discov. 2016;6(11):1248-1257.

75. Rizo A, Horton SJ, Olthof S, et al. BMI1 collaborates with BCR-ABL in leukemic transformation of human CD34+ cells. Blood. 2010;116(22):4621-4630

76. Sengupta A, Ficker AM, Dunn SK, Madhu $\mathrm{M}$, Cancelas JA. Bmi1 reprograms CML Blymphoid progenitors to become B-ALLinitiating cells. Blood. 2012;119(2):494-502.

77. Behzad MM, Shahrabi S, Jaseb K, et al Aberrant DNA methylation in chronic myeloid leukemia: cell fate control, prognosis, and therapeutic response. Biochem
Genet. 2018;56(3):149-175.

78. Heller G, Topakian T, Altenberger C, et al. Next-generation sequencing identifies major DNA methylation changes during progression of $\mathrm{Ph}+$ chronic myeloid leukemia. Leukemia. 2016;30(9):1861-1868

79. Mancini M, Velikovic N, Leo E, et al. Cytoplasmatic compartmentalization by Bcr-Abl promotes TET2 loss-of-function in chronic myeloid leukemia. J Cell Biochem. 2012;113(8):2765-2774

80. Amabile G, Di Ruscio A, Muller F, et al. Dissecting the role of aberrant DNA methylation in human leukaemia. Nat Commun. 2015;6:7091.

81. Issa JP, Gharibyan V, Cortes J, et al. Phase II study of low-dose decitabine in patients with chronic myelogenous leukemia resistant to imatinib mesylate. I Clin Oncol. 2005;23(17):3948-3956.

82. Visvader JE. Cells of origin in cancer Nature. 2011:469(7330):314-322.

83. Huntly BJ, Shigematsu H, Deguchi K, et al. MOZ-TIF2, but not BCR-ABL, confers properties of leukemic stem cells to committed murine hematopoietic progenitors. Cancer Cell. 2004;6(6):587-596.

84. Bose S, Deininger M, Gora-Tybor J, Goldman JM, Melo JV. The presence of typical and atypical BCR-ABL fusion genes in leukocytes of normal individuals: biologic significance and implications for the assessment of minimal residual disease. Blood. 1998:92(9):3362-3367.

85. Sparano JA, Gray RJ, Makower DF, et al Adjuvant chemotherapy guided by a 21 gene expression assay in breast cancer. $\mathrm{N}$ Engl J Med. 2018;379(2):111-121.

86. Ng SW, Mitchell A, Kennedy JA, et al. A 17gene stemness score for rapid determination of risk in acute leukaemia. Nature. 2016;540(7633):433-437.

87. Bill M, Nicolet D, Kohlschmidt J, et al. Mutations associated with a 17-gene leukemia stem cell score and the score's prognostic relevance in the context of the European LeukemiaNet classification of acute myeloid leukemia. Haematologica. 2020;105(3):721-729.

88. Shanmuganathan N, Pagani IS, Ross DM, et al. Early BCR-ABL1 kinetics are predictive of subsequent achievement of treatmentfree remission in chronic myeloid leukemia. Blood. 2021;137(9):1196-1207.

89. Radich JP, Larson R, Kantariian H, et al. Gene expression signature predicts deep molecular response (DMR) in chronic myeloid leukemia (CML): an exploratory biomarker analysis from ENESTnd [Abstract]. Blood. 2019;34;(Suppl_1):665.

90. Kwa M, Makris A, Esteva FJ. Clinical utility of gene-expression signatures in early stage breast cancer. Nat Rev Clin Oncol. 2017:14(10):595-610.

91. Teutsch SM, Bradley LA, Palomaki GE, et al. The Evaluation of Genomic Applications in Practice and Prevention (EGAPP) Initiative: methods of the EGAPP Working Group. Genet Med. 2009;11(1):3-14. 